February, 2013

OIQP-13-03

\title{
Non-planar operator mixing by Brauer representations
}

\author{
Yusuke Kimura1
}

\begin{abstract}
We study the action of the dilatation operator on the basis of local operators constructed from elements of the walled Brauer algebra, with non-planar corrections fully taken into account. We will see that the operator mixing can be neatly expressed in terms of the irreducible representations of the algebra. In particular we focus on a role of the integer that determines the number of boxes in the representations.
\end{abstract}

\section{Introduction}

In $4 \mathrm{D} \mathcal{N}=4$ Super Yang-Mills theory, conformal symmetry is a strong symmetry which we can use to restrict the form of correlation functions. As for two-point functions one has the form

$$
\left\langle O_{i}(x) O_{j}(y)\right\rangle=N_{i} \delta_{i j}(x-y)^{-\Delta_{i}}
$$

where $\Delta_{i}$ is the scaling dimension of the local operator. However, to obtain the scaling dimension in concrete cases we have to look for a good basis from the naive basis so that two-point functions have the diagonal form. (See [3, 4, 5, 6] for example). In other words the good operators are eigenstates of the dilatation operator with an eigenvalue $\Delta_{i}$ [1, 2].

Resolving the operator mixing problem needs a hard job in general, but as far as the planar limit is concerned, we have had a big progress since the discovery of an integrable structure underlying the theory [1, 7]. In the planar theory only single-trace operators come in the game, which are mapped to spin states. Considering only single-traces is justified by restricting our attention to operators whose classical dimension is much smaller than the size of the gauge group $N$. On the other hand, we need a new perspective if our focus is on large operators with a classical dimension comparable to $N$ or more, where huge combinatoric factors arising from summing up non-planar diagrams spoil the planar approximation. Consider a single-trace operator $\operatorname{tr}\left(X^{J}\right)$. It is given a good interpretation as a dual string object if $J \ll N$. In contrast, an issue arises if we consider a single-trace operator with $J \sim O(N)$. For example $\operatorname{tr}\left(X^{N+1}\right)$ can be expressed

\footnotetext{
${ }^{1}$ Okayama Institute for Quantum Physics (OIQP), Kyoyama 1-9-1, Kita-ku, Okayama, 700-0015, Japan; londonmileend@gmail.com
} 
as a linear combination of smaller operators. This implies that it should not correspond to an independent vertex operator in string theory. The equation to express $\operatorname{tr}\left(X^{N+1}\right)$ in terms of smaller operators can be obtained from the character of $S U(N)$ as $\chi_{R}(X)=0$, where $R=\left[1^{N+1}\right]$ is the anti-symmetric representation with $N+1$ boxes. This fact may encourage us to make use of Young diagrams for such large operators, and in fact Young diagrams can be used to count the number of large operators [8]. The constraint that Young diagrams should have no more than $N$ rows plays a role [9]. In the non-planar regime the operator corresponding to an independent vertex operator of string theory is a linear combination of multi-trace operators. Such large operators are considered to be dual to giant gravitons or geometries [10, 11, 12,

The finite $N$ issue for multi-matrix can also be understood in terms of Young diagrams. Some complete sets of local gauge invariant operators were constructed which are built from some kinds of fields [13, 14, 15, 16, 17]. They are labelled by a set of Young diagrams, and their notable property is that the operators have diagonal two-point functions at free theory, which are exact with respect to $N$. Such diagonal bases can be a good starting point to study the operator mixing problem for large operators. There have been some studies along this line to find that the operator mixing is neatly handled in terms of Young diagrams [18, 19, 20, 21, 22, 23, 24, 25, 26, 27, 28, 29, 30, 31, 32]. See [2, 33, 34, 35] for former approaches to non-planar corrections.

In this paper, we study the action of the dilatation operator on the Brauer basis introduced in [13. The basis has some labels, and one of the labels is an irreducible representation of the walled Brauer algebra $B_{N}(m, n)$ [36, 37, 38], which is given by a bi-partition,

$$
\gamma=\left(\gamma_{+}, \gamma_{-}\right)
$$

Hert 2

$$
\gamma_{+} \vdash(m-k), \quad \gamma_{-} \vdash(n-k),
$$

where $k$ is an integer obeying $0 \leq k \leq \min (m, n)$. We also denote it by $\gamma=\left(\gamma_{+}, \gamma_{-}, k\right)$ to emphasise what $k$ it has because we will pay attention to a role of the integer $k 3$ We will show how the two operators get mixed under the action of the dilatation operator. Particularly we will indicate the mixing condition for $k$. The mixing matrix we will obtain is expressed in terms of representation date and is very constrained. In other words, the mixing is very restricted on the representations. It would be a universal property for the diagonal bases labelled by a set of Young diagrams.

The paper is organised as follows. In section 2, we will study the one-loop dilatation operator on the Brauer basis. We follow the idea in [25] used to evaluate the dilatation operator on the restricted Schur basis. In section 3, we will write down the mixing condition in terms of the Brauer representations. In section 4, 5, we provide with the same study for two-loop. In section 6. the action of more general differential operators are studied. Section 7 is given for summary and discussions. Appendix $\mathrm{A}$ is for basic things of Brauer bases.

\footnotetext{
${ }^{2} R \vdash m$ is a shorthand notation to express that the Young diagram $R$ contains $m$ boxes.

${ }^{3}$ On a personal note the reason we focus on $k$ is as follows. For a class of $1 / 4$ BPS operators constructed in [22], a dual meaning of the integer $k$ was conjectured in the context of the correspondence with the $1 / 4$ BPS geometries in [39]. In our previous paper [40] we have studied correlation functions of Brauer operators and observed that correlation functions may be classified in terms of the integer $k$.
} 


\section{One-loop dilatation operator on Brauer basis}

The operator is

$$
O_{A, i j}^{\gamma}(X, Y)=t r_{m, n}\left(Q_{A, i j}^{\gamma} X^{\otimes m} \otimes Y^{T \otimes n}\right) .
$$

See appendix A for a detailed explanation.

The one-loop dilatation operator is given by $\hat{H}_{2}=-\operatorname{tr}\left([X, Y]\left[\partial_{X}, \partial_{Y}\right]\right)[2$, which acts on the Brauer basis as

$$
\begin{aligned}
\hat{H}_{2} O_{A, i j}^{\gamma} & =-m n t r_{m, n}\left(\left[Q_{A, i j}^{\gamma}, C_{m n}\right] X^{\otimes m-1} \otimes[X, Y] \otimes Y^{T \otimes n-1} \otimes 1\right) \\
& =-m n \sum_{r, k l} \frac{1}{d_{r}} \chi_{r, k l}^{\gamma}\left(\left[Q_{A, i j}^{\gamma}, C_{m n}\right]\right) t r_{m, n}\left(Q_{r, k l}^{\gamma} X^{\otimes m-1} \otimes[X, Y] \otimes Y^{T \otimes n-1} \otimes 1\right),
\end{aligned}
$$

where $r$ is an irreducible representation of the group algebra of $S_{m-1} \times S_{1} \times S_{n-1} \times S_{1}$. To obtain the second line we have used the inverse formula (A.12). The contraction is denoted by $C_{m n}$, which acts on the $m$-th slot of $X^{\otimes m}$ and the $n$-th slot of $Y^{T \otimes n}$.

We now recall the result of [22], where it was shown that the following operator

$$
O^{\gamma}(X, Y)=t r_{m, n}\left(P^{\gamma} X^{\otimes m} \otimes Y^{T \otimes n}\right),
$$

where $P^{\gamma}=\sum_{A, i} Q_{A, i i}^{\gamma}$ is the projection operator associated with an irreducible representation $\gamma$, is in the kernel of the dilatation operator. The result is very manifest in the form of (5) because of $\left[P^{\gamma}, C_{m n}\right]=0$.

Following the strategy in [25], we will expand (5) in terms of the basis (44). The readers who are not interested in the derivation can jump over to (17). Expressing the elements in $B_{N}(m, n)$ in terms of $B_{N}(m, n-1)$ as in (B.1), we haves

$$
\begin{aligned}
& t r_{m, n}\left(Q_{r, k l}^{\gamma} X^{\otimes m-1} \otimes[X, Y] \otimes Y^{T \otimes n-1} \otimes 1\right) \\
= & t^{\gamma} \sum_{b \in B_{N}(m, n)} \chi_{r, l k}^{\gamma}\left(b^{*}\right) t r_{m, n}\left(b X^{\otimes m-1} \otimes[X, Y] \otimes Y^{T \otimes n-1} \otimes 1\right) \\
= & t^{\gamma} \sum_{c \in B_{N}(m, n-1)} \chi_{r, l k}^{\gamma}\left(O_{c}^{*}\right) t r_{m, n-1}\left(c X^{\otimes m-1} \otimes[X, Y] \otimes Y^{T \otimes n-1}\right),
\end{aligned}
$$

where

$$
O_{c}^{*}:=N c^{*}+\sum_{i=1}^{n-1}((\bar{i}, \bar{n}) c)^{*}+\sum_{k=1}^{m}\left(c C_{k n}\right)^{*} .
$$

\footnotetext{
${ }^{4}$ We have the following equations for $c \in B_{N}(m, n-1)$;

$$
\begin{aligned}
& t r_{m, n}\left(c X^{\otimes m-1} \otimes[X, Y] \otimes Y^{T \otimes n-1} \otimes 1\right)=N t_{m, n-1}\left(c X^{\otimes m-1} \otimes[X, Y] \otimes Y^{T \otimes n-1}\right), \\
& t r_{m, n}\left((\bar{i}, \bar{n}) c X^{\otimes m-1} \otimes[X, Y] \otimes Y^{T \otimes n-1} \otimes 1\right)=t r_{m, n-1}\left(c X^{\otimes m-1} \otimes[X, Y] \otimes Y^{T \otimes n-1}\right), \\
& t r_{m, n}\left(c C_{k n} X^{\otimes m-1} \otimes[X, Y] \otimes Y^{T \otimes n-1} \otimes 1\right)=t r_{m, n-1}\left(c X^{\otimes m-1} \otimes[X, Y] \otimes Y^{T \otimes n-1}\right),
\end{aligned}
$$
}

where $i=1, \cdots, n-1$ and $k=1, \cdots, m$. 
The first equality in (8) comes from the definition of $Q_{r, k l}^{\gamma}$ (see (A.6)). Note that $c^{*}$ is not an element in $B_{N}(m, n-1)$ but an element in $B_{N}(m, n)$. Thanks to the formula (B.10) we find in appendix B, (8) can be rewritten as

$$
t^{\gamma} \sum_{c \in B_{N}(m, n-1)} \chi_{r, l k}^{\gamma}\left(c^{\tilde{*}}\right) t r_{m, n-1}\left(c X^{\otimes m-1} \otimes[X, Y] \otimes Y^{T \otimes n-1}\right) .
$$

Here $c^{\tilde{*}}$ is an element in $B_{N}(m, n-1)$. Using the following formula

$$
t r_{m, n-1}\left(c X^{\otimes m-1} \otimes[X, Y] \otimes Y^{T \otimes n-1}\right)=t r_{m, n}\left(\left[C_{m n}, c\right] X^{\otimes m} \otimes Y^{T \otimes n}\right)
$$

for $c \in B_{N}(m, n-1)$ and the inverse formula (A.9), we can expand the action of the one-loop dilatation operator in terms of the basis as

$$
\hat{H}_{2} O_{A, i j}^{\gamma}=\sum_{\gamma^{\prime}, A^{\prime}, p q} M_{(\gamma, A, i j)}^{\left(\gamma^{\prime}, A^{\prime}, p q\right)} O_{A^{\prime}, p q}^{\gamma^{\prime}}
$$

where the mixing matrix is

$$
M_{(\gamma, A, i j)}^{\left(\gamma^{\prime}, A^{\prime}, p q\right)}=-m n \sum_{r, k l} \frac{1}{d_{r}} \chi_{r, k l}^{\gamma}\left(\left[Q_{A, i j}^{\gamma}, C_{m n}\right]\right) t^{\gamma} \sum_{c \in B_{N}(m, n-1)} \chi_{r, l k}^{\gamma}\left(c^{\tilde{*}}\right) \frac{1}{d_{A^{\prime}}} \chi_{A^{\prime}, p q}^{\gamma^{\prime}}\left(\left[C_{m n}, c\right]\right) .
$$

Making use of the formula (A.13) 5, the two characters can be combined to give

$$
M_{(\gamma, A, i j)}^{\left(\gamma^{\prime}, A^{\prime}, p q\right)}=-m n t^{\gamma} \sum_{c \in B_{N}(m, n-1)} \chi^{\gamma}\left(\left[Q_{A, i j}^{\gamma}, C_{m n}\right] c^{\tilde{*}}\right) \frac{1}{d_{A^{\prime}}} \chi_{A^{\prime}, p q}^{\gamma^{\prime}}\left(\left[C_{m n}, c\right]\right) .
$$

We next perform the sum over $c$ using the orthogonality relation of the algebra. In order to use the orthogonality 6 for $B_{N}(m, n-1)$ we have to consider the restriction from $B_{N}(m, n)$ to a subalgebra $B_{N}(m, n-1)$. Let $\gamma_{1}$ be one of the irreducible representations that appear in $\gamma$ upon restricting $B_{N}(m, n)$ to $B_{N}(m, n-1)$, and $\gamma_{1}^{\prime}$ likewise. Then the sum can be evaluated in the following way using intertwiners

$$
\begin{aligned}
& \sum_{c \in B_{N}(m, n-1)} \chi^{\gamma}\left(\left[Q_{A, i j}^{\gamma}, C_{m n}\right] c^{\tilde{*}}\right) \chi_{A^{\prime}, p q}^{\gamma^{\prime}}\left(\left[C_{m n}, c\right]\right) \\
= & \sum_{c \in B_{N}(m, n-1)} \Gamma^{\gamma}\left(\left[Q_{A, i j}^{\gamma}, C_{m n}\right]\right)_{b a} \Gamma^{\gamma}\left(c^{\tilde{*}}\right)_{a b} \Gamma^{\gamma^{\prime}}\left(\left[Q_{A^{\prime}, q p}^{\gamma^{\prime}}, C_{m n}\right]\right)_{d c} \Gamma^{\gamma^{\prime}}(c)_{c d} \\
= & \sum_{\gamma_{1}, \gamma_{1}^{\prime}} \frac{1}{t^{\gamma}} \operatorname{tr}\left(\Gamma^{\gamma}\left(\left[Q_{A, i j}^{\gamma}, C_{m n}\right]\right) I_{\gamma_{1} \gamma_{1}^{\prime}} \Gamma^{\gamma^{\prime}}\left(\left[Q_{A^{\prime}, q p}^{\gamma^{\prime}}, C_{m n}\right]\right) I_{\gamma_{1}^{\prime} \gamma_{1}}\right) .
\end{aligned}
$$

$I_{\gamma_{1} \gamma_{1}^{\prime}}$ is an intertwiner that maps from $\gamma_{1}$ to $\gamma_{1}^{\prime}$, and it is non-zero if $\gamma_{1}$ and $\gamma_{1}^{\prime}$ are the same shape. See appendix B in [25] or appendix D in [27] for more details on the intertwiners.

\footnotetext{
${ }^{5}$ Note that $\left[Q_{A, i j}^{\gamma}, C_{m n}\right]$ commutes with any elements in $S_{m-1} \times S_{1} \times S_{n-1} \times S_{1}$.

${ }^{6}$ The orthogonality relation of the Brauer algebra is given by

$$
\sum_{c \in B_{N}(m, n-1)} \Gamma^{\left(\gamma_{1}\right)}(c)_{\alpha \beta} \Gamma^{\left(\gamma_{1}^{\prime}\right)}\left(c^{\tilde{*}}\right)_{\gamma \delta}=\frac{1}{t^{\gamma_{1}}} \delta_{\alpha \delta} \delta_{\beta \gamma} \delta^{\gamma_{1} \gamma_{1}^{\prime}}
$$

where $\gamma_{1}, \gamma_{1}^{\prime}$ are irreducible representations of $B_{N}(m, n-1)$.
} 
Then the final form of our mixing matrix is

$$
M_{(\gamma, A, i j)}^{\left(\gamma^{\prime}, A^{\prime}, p q\right)}=-m n \frac{1}{d_{A^{\prime}}} \sum_{\gamma_{1}, \gamma_{1}^{\prime}} \frac{t^{\gamma}}{t^{\gamma_{1}}} \chi^{\gamma}\left(\left[Q_{A, i j}^{\gamma}, C_{m n}\right] I_{\gamma_{1} \gamma_{1}^{\prime}}\left[Q_{A^{\prime}, q p}^{\gamma^{\prime}}, C_{m n}\right] I_{\gamma_{1}^{\prime} \gamma_{1}}\right) .
$$

We have obtained a very similar form to the case of restricted Schur basis. The $N$-dependence is exact.

We find that the BPS operators ([6) do not appear even in the image of the dilatation operator:

$$
\begin{aligned}
\left\langle O^{\gamma^{\prime \prime}} \hat{H}_{2} O_{A, i j}^{\gamma}\right\rangle & =\sum_{p} \sum_{A^{\prime \prime}} M_{(\gamma, A, i j)}^{\left(\gamma^{\prime \prime}, A^{\prime \prime}, p p\right)} m ! n ! d_{A^{\prime \prime}} t^{\gamma^{\prime \prime}} \\
& \sim \chi^{\gamma}\left(\cdots\left[P^{\gamma^{\prime \prime}}, C_{m n}\right] \cdots\right)=0,
\end{aligned}
$$

where we have used the two-point function (A.5). Therefore they are completely separated from the others.

The first line of (5) may be written as

$$
\begin{aligned}
\hat{H}_{2} O_{A, i j}^{\gamma}= & -m n t r_{m, n}\left(Q_{A, i j}^{\gamma} C_{m n} X^{\otimes m-1} \otimes X Y \otimes Y^{T \otimes n-1} \otimes 1\right) \\
& +m n t r_{m, n}\left(Q_{A, i j}^{\gamma} C_{m n} X^{\otimes m} \otimes Y^{T \otimes n}\right) \\
& +m n t r_{m, n}\left(C_{m n} Q_{A, i j}^{\gamma} X^{\otimes m} \otimes Y^{T \otimes n}\right) \\
& -m n t r_{m, n}\left(C_{m n} Q_{A, i j}^{\gamma} X^{\otimes m-1} \otimes Y X \otimes Y^{T \otimes n-1} \otimes 1\right) .
\end{aligned}
$$

This leads to another form of the mixing matrix:

$$
\begin{aligned}
M_{(\gamma, A, i j)}^{\left(\gamma^{\prime}, A^{\prime}, p q\right)}= & -m n \frac{1}{d_{A^{\prime}}} \sum_{\gamma_{1}, \gamma_{1}^{\prime}} \frac{t^{\gamma}}{t_{1}} \chi^{\gamma}\left(Q_{A, i j}^{\gamma} C_{m n} I_{\gamma_{1} \gamma_{1}^{\prime}} Q_{A^{\prime}, q p}^{\gamma^{\prime}} C_{m n} I_{\gamma_{1}^{\prime} \gamma_{1}}\right) \\
& -m n \frac{1}{d_{A^{\prime}}} \sum_{\gamma_{1}, \gamma_{1}^{\prime}} \frac{t^{\gamma}}{t^{\gamma_{1}}} \chi^{\gamma}\left(C_{m n} Q_{A, i j}^{\gamma} I_{\gamma_{1} \gamma_{1}^{\prime}} C_{m n} Q_{A^{\prime}, q p}^{\gamma^{\prime}} I_{\gamma_{1}^{\prime} \gamma_{1}}\right) \\
& +\delta_{\gamma}^{\gamma^{\prime}} \delta_{A}^{A^{\prime}} \delta_{i}^{p} m n \frac{1}{d_{A}} \chi_{A, j q}^{\gamma}\left(C_{m n}\right)+\delta_{\gamma}^{\gamma^{\prime}} \delta_{A}^{A^{\prime}} \delta_{j}^{q} m n \frac{1}{d_{A}} \chi_{A, p i}^{\gamma}\left(C_{m n}\right) .
\end{aligned}
$$

\section{One-loop mixing}

The one-loop mixing matrix we have obtained can be shown to be in fact almost diagonal. The necessary condition to have a non-zero mixing is that $\gamma_{1}$ and $\gamma_{1}^{\prime}$ are the same bi-partition. In this section we will translate this condition into a condition between $\gamma$ and $\gamma^{\prime}$.

The restriction of $B_{N}(m, n)$ to $B_{N}(m, n-1)$ was studied in [40]. An irreducible representation $\gamma_{1}$ in $B_{N}(m, n-1)$ can appear in an irreducible representation $\gamma$ in $B_{N}(m, n)$ if the following quantity is non-zero [37]

$$
M_{\gamma \rightarrow \gamma_{1}}=\sum_{\delta, \zeta} g\left(\delta, \gamma_{+} ; \gamma_{1+}\right) g(\delta, \zeta ;[1]) g\left(\zeta, \gamma_{1-} ; \gamma_{-}\right)
$$

where $g(\alpha, \beta ; \gamma)$ is the Littlewood-Richardson coefficient. We now introduce a new notation

$$
R=S^{(+1)}
$$


to denote that $R$ is a Young diagram obtained from $S$ by adding a box. We also denote it by $S=R^{(-1)}$. For instance $R^{(-1)}=R^{\prime(-1)}$ means that $R$ and $R^{\prime}$ are related each other by moving a single box. In terms of this new notation, there are two cases for $M_{\gamma \rightarrow \gamma_{1}}$ to be non-zero:

$$
\gamma_{+}=\gamma_{1+}, \quad \gamma_{-}=\gamma_{1-}^{(+1)}
$$

which is the case $(\delta, \zeta)=(\emptyset,[1])$, and

$$
\gamma_{+}^{(+1)}=\gamma_{1+}, \quad \gamma_{-}=\gamma_{1-},
$$

which is the case $(\delta, \zeta)=([1], \emptyset)$. Introducing $\Delta=k-k_{1}$, the first case is $\Delta=0$ while the second is $\Delta=1$.

We find that to get a non-zero mixing element we need one of the following conditions between $\gamma$ and $\gamma^{\prime}$ :

- $\left(\Delta, \Delta^{\prime}\right)=(0,0)$

$$
\gamma_{+}=\gamma_{+}^{\prime}, \quad \gamma_{-}^{(-1)}=\gamma_{-}^{\prime(-1)} \quad\left[k=k^{\prime}\right]
$$

- $\left(\Delta, \Delta^{\prime}\right)=(1,1)$

$$
\gamma_{+}^{(+1)}=\gamma_{+}^{\prime(+1)}, \quad \gamma_{-}=\gamma_{-}^{\prime} \quad\left[k=k^{\prime}\right]
$$

- $\left(\Delta, \Delta^{\prime}\right)=(0,1)$

$$
\gamma_{+}=\gamma_{+}^{\prime(+1)}, \quad \gamma_{-}^{(-1)}=\gamma_{-}^{\prime} \quad\left[k=k^{\prime}-1\right]
$$

- $\left(\Delta, \Delta^{\prime}\right)=(1,0)$

$$
\gamma_{+}^{(+1)}=\gamma_{+}^{\prime}, \quad \gamma_{-}=\gamma_{-}^{\prime(-1)} \quad\left[k=k^{\prime}+1\right]
$$

The one-loop dilatation operator induces an interaction between two sets of Young diagrams that are related by moving a box. In this sense, we say that only "nearest-neighbour" Young diagrams interact each other at one-loop. This seems to be an interesting extension of the planar case, where the one-loop dilatation operator was a nearest-neighbour interaction on spin states. This kind of nearest-neighbour interactions on Young diagrams have been already seen on the other bases [20, 25]. We suspect that in our case the integer $k$ is a convenient index to know if two operators are nearest-neighbours.

\section{Two-loop dilatation operator on Brauer basis}

In this section, we will evaluate the action of the two-loop dilatation operator on the Brauer basis. The two-loop dilatation operator on the restricted Schur basis was evaluated in [30].

The two-loop dilatation operator was given in [2] as

$$
\begin{aligned}
\hat{H}_{4}= & -\operatorname{tr}\left(:\left[[Y, X], \partial_{X}\right]\left[\left[\partial_{Y}, \partial_{X}\right], X\right]:\right)-\operatorname{tr}\left(:\left[[Y, X], \partial_{Y}\right]\left[\left[\partial_{Y}, \partial_{X}\right], Y\right]:\right) \\
& -\operatorname{tr}\left(:\left[[Y, X], T_{a}\right]\left[\left[\partial_{Y}, \partial_{X}\right], T_{a}\right]:\right) .
\end{aligned}
$$


The last term is identical to the one-loop dilatation operator. Because the second term is obtained by exchanging $X$ and $Y$ in the first term, we will study only the first term.

After some straightforward calculation, which we will show in appendix C, we obtain

$$
\begin{aligned}
& \frac{1}{m(m-1) n} \operatorname{tr}\left(:\left[[Y, X], \partial_{X}\right]\left[\left[\partial_{Y}, \partial_{X}\right], X\right]:\right) O_{A, i j}^{\gamma} \\
= & t r_{m, n}\left(\left[C_{m n}(m-1, m), Q_{A, i j}^{\gamma}\right] X^{\otimes m-2} \otimes X[Y, X] \otimes 1 \otimes Y^{T \otimes n-1} \otimes 1\right) \\
& -t r_{m, n}\left(\left[(m-1, m) C_{m n}, Q_{A, i j}^{\gamma}\right] X^{\otimes m-1} \otimes[Y, X] \otimes Y^{T \otimes n-1} \otimes 1\right) \\
& -t r_{m, n}\left(\left[C_{m n}(m-1, m), Q_{A, i j}^{\gamma}\right] X^{\otimes m-1} \otimes[Y, X] \otimes Y^{T \otimes n-1} \otimes 1\right) \\
& +t r_{m, n}\left(\left[(m-1, m) C_{m n}, Q_{A, i j}^{\gamma}\right] X^{\otimes m-2} \otimes[Y, X] X \otimes 1 \otimes Y^{T \otimes n-1} \otimes 1\right) .
\end{aligned}
$$

The second and the third term above are similar to the one-loop result. We further rewrite the first term and the forth term, which we need the reduction formula twice.

Note that in the form it is pretty manifest that the operators (6) are BPS at two-loop. It is interesting to observe that $Q_{A, i j}^{\gamma}$ always appears in the form $\left[Q_{A, i j}^{\gamma}, b\right]$ with an element $b$ in the algebra.

We now concretely show how the first term in (30) can be further rewritten. Using the inverse formula it can be expanded in terms of

$$
t r_{m, n}\left(Q_{s, k l}^{\gamma} X^{\otimes m-2} \otimes X[Y, X] \otimes 1 \otimes Y^{T \otimes n-1} \otimes 1\right)
$$

where $s$ is an irreducible representation of the group algebra of $S_{m-2} \times S_{1} \times S_{1} \times S_{n-1} \times S_{1}$. After using the formula given in appendix $\mathrm{B}$ twice, the above operator can be rewritten as

$$
\begin{aligned}
& t^{\gamma} \sum_{b \in B_{N}(m, n)} \chi_{s, l k}^{\gamma}\left(b^{*}\right) t r_{m, n}\left(b X^{\otimes m-2} \otimes X[Y, X] \otimes 1 \otimes Y^{T \otimes n-1} \otimes 1\right) \\
= & t^{\gamma} \sum_{c \in B_{N}(m-1, n-1)} \chi_{s, l k}^{\gamma}\left(c^{\tilde{*}}\right) t r_{m-1, n-1}\left(c X^{\otimes m-2} \otimes X[Y, X] \otimes Y^{T \otimes n-1}\right) .
\end{aligned}
$$

Here the dual element $c^{\tilde{*}}$ is defined in $B_{N}(m-1, n-1)$. We can use the following equations for $c \in B_{N}(m-1, n-1)$ :

$$
\begin{aligned}
t r_{m-1, n-1}\left(c X^{\otimes m-2} \otimes X Y X \otimes Y^{T \otimes n-1}\right) & =t r_{m, n}\left((m-1, m) C_{m n} c X^{\otimes m} \otimes Y^{T \otimes n}\right), \\
t r_{m-1, n-1}\left(c X^{\otimes m-2} \otimes X X Y \otimes Y^{T \otimes n-1}\right) & =t r_{m, n}\left(C_{m n}(m-1, m) c X^{\otimes m} \otimes Y^{T \otimes n}\right), \\
t r_{m-1, n-1}\left(c X^{\otimes m-2} \otimes Y X X \otimes Y^{T \otimes n-1}\right) & =t r_{m, n}\left(c(m-1, m) C_{m n} X^{\otimes m} \otimes Y^{T \otimes n}\right) .
\end{aligned}
$$

Note that if we use $\left[c, C_{m n}\right]=0$ for $c \in B_{N}(m-1, n-1)$ and $(m-1, m) X^{\otimes m}=X^{\otimes m}(m-1, m)$, there are several equivalent expressions like

$$
t r_{m, n}\left((m-1, m) C_{m n} c X^{\otimes m} \otimes Y^{T \otimes n}\right)=t r_{m, n}\left(c C_{m n}(m-1, m) X^{\otimes m} \otimes Y^{T \otimes n}\right) .
$$


Then the first term in (30) is

$$
\begin{aligned}
& t r_{m, n}\left(\left[C_{m n}(m-1, m), Q_{A, i j}^{\gamma}\right] X^{\otimes m-2} \otimes 1 \otimes X[Y, X] \otimes Y^{T \otimes n-1} \otimes 1\right) \\
= & \sum_{s, k l} \frac{1}{d_{s}} \chi_{s, k l}^{\gamma}\left(\left[C_{m n}(m-1, m), Q_{A, i j}^{\gamma}\right]\right) t r_{m, n}\left(Q_{s, k l}^{\gamma} X^{\otimes m-2} \otimes 1 \otimes X[Y, X] \otimes Y^{T \otimes n-1} \otimes 1\right) \\
= & \sum_{s, k l} \frac{1}{d_{s}} \chi_{s, k l}^{\gamma}\left(\left[C_{m, n}(m-1, m), Q_{A, i j}^{\gamma}\right]\right) \\
& \times t^{\gamma} \sum_{c \in B(m-1, n-1)} \chi_{s, l k}^{\gamma}\left(c^{\tilde{*}}\right) t r_{m-1, n-1}\left(c X[Y, X] \otimes X^{\otimes m-2} \otimes Y^{T \otimes n-1}\right) \\
= & \sum_{s, k l} \frac{1}{d_{s}} \chi_{s, k l}^{\gamma}\left(\left[C_{m n}(m-1, m), Q_{A, i j}^{\gamma}\right]\right) \\
& \times t^{\gamma} \sum_{c \in B(m-1, n-1)} \chi_{s, l k}^{\gamma}\left(c^{\tilde{*}}\right) t r_{m, n}\left(\left[c, C_{m n}(m-1, m)\right] X^{\otimes m} \otimes Y^{T \otimes n}\right) \\
= & \sum_{s, k l} \frac{1}{d_{s}} \chi_{s, k l}^{\gamma}\left(\left[C_{m n}(m-1, m), Q_{A, i j}^{\gamma}\right]\right) t^{\gamma} \sum_{c \in B(m-1, n-1)} \chi_{s, l k}^{\gamma}\left(c^{\tilde{*}}\right) \\
& \times \sum_{\gamma^{\prime}, A^{\prime}, p q} \frac{1}{d_{A^{\prime}}} \chi_{A^{\prime}, p q}^{\gamma^{\prime}}\left(\left[c, C_{m n}(m-1, m)\right]\right) t r_{m, n}\left(Q_{A^{\prime}, p q}^{\gamma^{\prime}} \otimes X^{\otimes m} \otimes Y^{T \otimes n}\right) .
\end{aligned}
$$

Making use of (A.13) and performing the sum of $c \in B_{N}(m-1, n-1)$, we have

$$
\hat{H}_{4} O_{A, i j}^{\gamma}=M_{(\gamma, A, i j)}^{\left(\gamma^{\prime}, A^{\prime}, p q\right)} O_{A^{\prime}, p q}^{\gamma^{\prime}},
$$

where the contribution from the first term in $\left(\underline{30)}\right.$ to $M_{(\gamma, A, i j)}^{\left(\gamma^{\prime}, A^{\prime}, p q\right)}$ is

$$
m(m-1) n \sum_{\gamma_{2}, \gamma_{2}^{\prime}} \frac{t^{\gamma}}{t^{\gamma_{2}}} \frac{1}{d_{A^{\prime}}} \chi^{\gamma}\left(\left[C_{m n}(m-1, m), Q_{A, i j}^{\gamma}\right] I_{\gamma_{2} \gamma_{2}^{\prime}}\left[C_{m n}(m-1, m), Q_{A^{\prime}, q p}^{\gamma^{\prime}}\right] I_{\gamma_{2}^{\prime} \gamma_{2}}\right)
$$

where $\gamma_{2}$ and $\gamma_{2}^{\prime}$ are irreducible representations of $B_{N}(m-1, n-1)$. Likewise the forth term in (30) gives

$$
m(m-1) n \sum_{\gamma_{2}, \gamma_{2}^{\prime}} \frac{t^{\gamma}}{t^{\gamma_{2}}} \frac{1}{d_{A^{\prime}}} \chi^{\gamma}\left(\left[(m-1, m) C_{m n}, Q_{A, i j}^{\gamma}\right] I_{\gamma_{2} \gamma_{2}^{\prime}}\left[(m-1, m) C_{m n}, Q_{A^{\prime}, q p}^{\gamma^{\prime}}\right] I_{\gamma_{2}^{\prime} \gamma_{2}}\right) .
$$

From these expressions we find that the BPS operators (6) do not appear in the image under the two-loop dilatation operator.

\section{Two-loop mixing}

The necessary condition for the two-loop mixing matrix to be non-zero is that $\gamma_{2}$ and $\gamma_{2}^{\prime}$ are the same shape. The mixing structure is determined by the restriction $B_{N}(m, n) \rightarrow B_{N}(m-1, n-$ $1) \times B_{N}(1,1)$. The number of times $\gamma_{2}$ appears in an irreducible representation $\gamma$ is counted by

$$
\begin{gathered}
M_{\gamma_{2}, \gamma_{3}}^{\gamma}=\sum_{\rho, \zeta, \theta, \kappa}\left(\sum_{\delta} g\left(\delta, \rho ; \gamma_{2+}\right) g\left(\delta, \zeta ; \gamma_{3-}\right)\right)\left(\sum_{\epsilon} g\left(\epsilon, \theta ; \gamma_{2-}\right) g\left(\epsilon, \kappa ; \gamma_{3+}\right)\right) \\
\times g\left(\rho, \kappa ; \gamma_{+}\right) g\left(\zeta, \theta ; \gamma_{-}\right),
\end{gathered}
$$


where we have denoted an irreducible representation of $B_{N}(1,1)$ by $\gamma_{3}$. There are two irreducible representations in $B_{N}(1,1) ; \gamma_{3}=\left([1],[1], k_{3}=0\right)$ or $\gamma_{3}=\left(\emptyset, \emptyset, k_{3}=1\right)$. For the former case, we have

$M_{\gamma_{2}, \gamma_{3}\left(k_{3}=0\right)}^{\gamma}=\sum_{\rho, \zeta, \theta, \kappa}\left(\sum_{\delta} g\left(\delta, \rho ; \gamma_{2+}\right) g(\delta, \zeta ;[1])\right)\left(\sum_{\epsilon} g\left(\epsilon, \theta ; \gamma_{2-}\right) g(\epsilon, \kappa ;[1])\right) g\left(\rho, \kappa ; \gamma_{+}\right) g\left(\zeta, \theta ; \gamma_{-}\right)$,

while for the latter case we have

$$
\begin{aligned}
M_{\gamma_{2}, \gamma_{3}\left(k_{3}=1\right)}^{\gamma} & =\sum_{\rho, \zeta, \theta, \kappa}\left(\sum_{\delta} g\left(\delta, \rho ; \gamma_{2+}\right) g(\delta, \zeta ; \emptyset)\right)\left(\sum_{\epsilon} g\left(\epsilon, \theta ; \gamma_{2-}\right) g(\epsilon, \kappa ; \emptyset)\right) g\left(\rho, \kappa ; \gamma_{+}\right) g\left(\zeta, \theta ; \gamma_{-}\right) \\
& =g\left(\gamma_{2+}, \emptyset ; \gamma_{+}\right) g\left(\emptyset, \gamma_{2-} ; \gamma_{-}\right) .
\end{aligned}
$$

In these cases the $M_{\gamma_{2}, \gamma_{3}}^{\gamma}$ takes 0 or 1 .

We find that there are five cases the $M_{\gamma_{2}, \gamma_{3}}^{\gamma}$ takes the non-zero value. Introducing $\Delta=$ $k-k_{2}-k_{3}$, we list the five cases below:

- $\gamma_{2+}=\gamma_{+}^{(-1)}, \gamma_{2-}=\gamma_{-}^{(-1)} \quad\left[k_{3}=0, k=k_{2}, \Delta=0\right]$,

- $\gamma_{2+}=\gamma_{+}, \gamma_{2-}=\gamma_{-} \quad\left[k_{3}=1, k=k_{2}+1, \Delta=0\right]$,

- $\gamma_{2+}=\gamma_{+}, \gamma_{2-}^{(-1)}=\gamma_{-}^{(-1)} \quad\left[k_{3}=0, k=k_{2}+1, \Delta=1\right]$,

- $\gamma_{2+}^{(-1)}=\gamma_{+}^{(-1)}, \gamma_{2-}=\gamma_{-} \quad\left[k_{3}=0, k=k_{2}+1, \Delta=1\right]$

- $\gamma_{2+}=\gamma_{+}^{(+1)}, \gamma_{2-}=\gamma_{-}^{(+1)} \quad\left[k_{3}=0, k=k_{2}+2, \Delta=2\right]$.

They correspond to $(\delta, \zeta, \epsilon, \kappa)=(0,[1], 0,[1]),(0,0,0,0),(0,[1],[1], 0),([1], 0,0,[1]),([1], 0,[1], 0)$ respectively.

Because there are five cases for the restriction from $\gamma^{\prime}$ to $\gamma_{2}^{\prime}$ as well, we have twenty-five cases $\gamma$ and $\gamma^{\prime}$ interact each other in which $k$ and $k^{\prime}$ are related by $k^{\prime}=k, k \pm 1, k \pm 2$. We will show some of them:

- $\left(\Delta, \Delta^{\prime}\right)=(0,0),\left(k_{3}, k_{3}^{\prime}\right)=(0,0)$

$$
\gamma_{+}^{(-1)}=\gamma_{+}^{\prime(-1)}, \quad \gamma_{-}^{(-1)}=\gamma_{-}^{\prime(-1)} \quad\left[k=k^{\prime}\right],
$$

- $\left(\Delta, \Delta^{\prime}\right)=(0,2),\left(k_{3}, k_{3}^{\prime}\right)=(1,0)$

$$
\gamma_{+}=\gamma_{+}^{\prime}{ }^{(+1)}, \quad \gamma_{-}=\gamma_{-}^{\prime(+1)} \quad\left[k=k^{\prime}-1\right],
$$

- $\left(\Delta, \Delta^{\prime}\right)=(2,0),\left(k_{3}, k_{3}^{\prime}\right)=(0,0)$

$$
\gamma_{+}^{(+1)}=\gamma_{+}^{\prime(-1)}, \quad \gamma_{-}^{(+1)}=\gamma_{-}^{\prime(-1)} \quad\left[k=k^{\prime}+2\right],
$$

- $\left(\Delta, \Delta^{\prime}\right)=(0,0),\left(k_{3}, k_{3}^{\prime}\right)=(1,1)$

$$
\gamma_{+}=\gamma_{+}^{\prime}, \quad \gamma_{-}=\gamma_{-}^{\prime} \quad\left[k=k^{\prime}\right],
$$

- $\left(\Delta, \Delta^{\prime}\right)=(1,1),\left(k_{3}, k_{3}^{\prime}\right)=(0,0)$

$$
\begin{aligned}
& \gamma_{+}=\gamma_{+}^{\prime}, \quad \gamma_{-}^{(-2)}=\gamma_{-}^{\prime}{ }^{(-2)} \quad\left[k=k^{\prime}\right], \\
& \gamma_{+}^{(-2)}=\gamma_{+}^{\prime}{ }^{(-2)}, \quad \gamma_{-}=\gamma_{-}^{\prime} \quad\left[k=k^{\prime}\right], \\
& \gamma_{+}^{(-1)}=\gamma_{+}^{\prime}{ }^{(-1)}, \quad \gamma_{-}^{(-1)}=\gamma_{-}^{\prime}{ }^{(-1)} \quad\left[k=k^{\prime}\right],
\end{aligned}
$$

where the last one appears twice. 


\section{More general differential operators}

In this section we will study the action of more general differential operators on the basis with focusing on the role of $k$. Let us first consider the differential operator $\operatorname{tr}\left(W \partial_{X} \partial_{X} \partial_{Y} \partial_{Y}\right)$ as an example, assuming that $W$ is made from two copies of $X$ and two copies of $Y$. The action on the basis can be evaluated as

$$
\begin{aligned}
& \operatorname{tr}\left(W \partial_{X} \partial_{X} \partial_{Y} \partial_{Y}\right) O_{A, i j}^{\gamma}(X, Y) \\
= & m(m-1) n(n-1) t r_{m, n}\left(Q_{A, i j}^{\gamma} C_{2,1}(12)(\overline{1} \overline{2}) W \otimes 1 \otimes X^{\otimes m-2} \otimes 1 \otimes 1 \otimes Y^{T \otimes n-2}\right) \\
= & m(m-1) n(n-1) \sum_{r, k l} \chi_{r, k l}^{\gamma}\left(Q_{A, i j}^{\gamma} C_{2,1}(12)(\overline{1} \overline{2})\right) \\
& \times t r_{m, n}\left(Q_{r, k l}^{\gamma} W \otimes 1 \otimes X^{\otimes m-2} \otimes 1 \otimes 1 \otimes Y^{T \otimes n-2}\right),
\end{aligned}
$$

where $r$ is an irreducible representation of the group algebra of $S_{m-2} \times S_{1} \times S_{1} \times S_{n-2} \times S_{1} \times S_{1}$.

The next step is to use the reduction rule three times, followed by taking the sum of $c \in$ $B_{N}(m-1, n-2)$ appearing in $Q_{r, k l}^{\gamma}$. Finally, we will have $\chi^{\gamma}\left(\cdots I_{\gamma_{2} \gamma_{2}^{\prime}} \cdots I_{\gamma_{2}^{\prime} \gamma_{2}}\right)$, where $\gamma_{2}$ and $\gamma_{2}^{\prime}$ are irreducible representations of $B_{N}(m-1, n-2)$. This leads to considering the restriction $B_{N}(m, n) \rightarrow B_{N}(m-1, n-2) \times B_{N}(1,2)$, which determines the mixing structure. Denoting an irreducible representation of $B_{N}(1,2)$ by $\gamma_{3}$, the necessary condition for the non-zero mixing is that the $M_{\gamma_{2}, \gamma_{3}}^{\gamma}$, which is given by the same form as (39), is non-zero. Denoting the number of boxes in a partition $\alpha$ by $n(\alpha)$, we find that $n(\delta)$ and $n(\zeta)$ take $0,1,2$, and $n(\epsilon)$ and $n(\kappa)$ take 0,1 . From the consistency, we have the relation

$$
n\left(\gamma_{+}\right)=n(\kappa)+n\left(\gamma_{2+}\right)-n(\delta)
$$

which implies

$$
k-k_{2}=0,1,2,3
$$

Therefore the necessary condition for non-zero mixing between two representations $\gamma$ and $\gamma^{\prime}$ is $k^{\prime}=k, k \pm 1, k \pm 2, k \pm 3$. Schematically writing, we have

$$
\operatorname{tr}\left(W \partial_{X} \partial_{X} \partial_{Y} \partial_{Y}\right) O^{\gamma(k)} \sim \sum_{k^{\prime}=k-3}^{k+3} a_{k^{\prime}} O^{\gamma^{\prime}\left(k^{\prime}\right)} .
$$

If we consider the action of a more general differential operator with $p \partial_{X}$ 's and $q \partial_{Y}$ 's, we have to consult the restriction $B_{N}(m, n) \rightarrow B_{N}(m-p+1, n-q) \times B_{N}(p-1, q)$. By a similar argument to the above case, we find that

$$
k-k_{2}=0,1,2, \cdots, p+q-1,
$$

which implies

$$
k^{\prime}=k, k \pm 1, \cdots, k \pm(p+q-1) .
$$




\section{$7 \quad$ Summary and discussions}

In this paper we have expressed the following mixing matrix in terms of Brauer representation data with keeping the $N$-dependence exact,

$$
\left\langle O_{A^{\prime}, i^{\prime} j^{\prime}}^{\gamma^{\prime}} \hat{H}_{2 l} O_{A, i j}^{\gamma}\right\rangle
$$

We have found that the mixing on the representations is highly restrictive. Such restricted mixing would be a universal property of the bases for which the free two-point function is diagonal.

In particular we have focused on the integer $k$ that determines the number of boxes in the irreducible representation $\gamma$ of the Brauer algebra. To get a non-zero mixing result, we need $k-k^{\prime}=0, \pm 1$ at one-loop and $k-k^{\prime}=0, \pm 1, \pm 2$ at two-loop. This mixing is reminiscent of 23]. It would be interesting to consider if the dilatation operator can be viewed as a lattice approximation to a second derivative on $k$. We also have the mixing for the Young diagrams $\left(\gamma_{+}\right.$, $\left.\gamma_{-}\right)$. The diagrams $\left(\gamma_{+}, \gamma_{-}\right)$encode the information of $k$ but if we consider a three-dimensional space spanned by $\gamma_{+}, \gamma_{-}$and $k$, we might get a good perspective to understand the mixing. In order to gain more understanding of a role of the integer $k$, it would also be nice to study the dilatation operator on the Brauer bases beyond $\mathrm{su}(2)$ sector [40, 41].

We have explicitly confirmed that the construction of some 1/4 BPS operators in [22] is valid at two-loop. We also saw that they do not appear in the image of the dilatation operator.

The next direction we should proceed to is to diagonalise the mixing matrix. Recently there have been an interesting progress in diagonalising the mixing matrix on the restricted Schur operators [25, 26, 27, 30, 32]. They have shown that the action of the dilatation operator reduces to systems of harmonic oscillators when the corners of the Young diagram are well-separated (displaced corner approximation). The idea underlying the approximation is to exploit a good set of conserved charges preserved by the dilatation operator. In the series of works, the charges encode open string configurations on giant gravitons [28, 29]. (Another approach can be seen in 42.) Finding good conserved charges is a nice pathway towards diagonalising the mixing

matrix. Because such conserved charges correspond to parameters of the dual physics, it would be helpful to conduct an analysis in the string/gravity side. In [39] we have studied a correspondence between 1/4 BPS operators and 1/4 BPS geometries, where it was shown that the geometries are characterised by an integer that has the same upper bound as the integer $k$ (: recall $k \leq$ $\min (m, n))$. This fact might be a clue to solve the operator mixing dual to geometries.

Acknowledgements: I would like to thank Robert de Mello Koch and Sanjaye Ramgoolam for helpful discussions. I also would like to thank Durham university. Discussions during the workshop "Symmetry and Geometry of Branes in String/M Theory" were useful.

\section{A Basic facts}

In this appendix, we summarise basic things of Brauer operators, which were proposed in [13] and further studied in [17, 22, 40, 43]. The operator we consider can be expressed by

$$
O_{A, i j}^{\gamma}(X, Y)=t r_{m, n}\left(Q_{A, i j}^{\gamma} X^{\otimes m} \otimes Y^{T \otimes n}\right),
$$

where $\gamma=\left(\gamma_{+}, \gamma_{-}\right)$is an irreducible representation of the walled Brauer algebra $B_{N}(m, n)$, and a pair of Young diagrams $A=(\alpha, \beta)$ is an irreducible representation of $S_{m} \times S_{n}$. The group 
algebra of $S_{m} \times S_{n}$ is a subalgebra of $B_{N}(m, n)$, and a representation $\gamma$ in general contains some representations of the group algebra of $S_{m} \times S_{n}$. When a particular representation of the group algebra of $S_{m} \times S_{n}$ appears more than once in the restriction, the labels $i, j$ specify which copy of the representation we consider. The multiplicity of the representation $A$ in a representation $\gamma$ is given by

$$
M_{A}^{\gamma}=\sum_{\delta \vdash k} g\left(\delta, \gamma_{+} ; \alpha\right) g\left(\delta, \gamma_{-} ; \beta\right),
$$

where $g\left(\delta, \gamma_{+} ; \alpha\right)$ is the Littlewood-Richardson coefficient. The labels on the operator are summarised as

$$
\begin{aligned}
& \gamma=\left(\gamma_{+}, \gamma_{-}\right), \quad \gamma_{+} \vdash(m-k), \quad \gamma_{-} \vdash(n-k) \\
& A=(\alpha, \beta), \quad \alpha \vdash m, \quad \beta \vdash n \\
& i, j=1, \cdots, M_{A}^{\gamma},
\end{aligned}
$$

where $k$ is an integer in the range $0 \leq k \leq \min (m, n)$. The representations should satisfy

$$
c_{1}\left(\gamma_{+}\right)+c_{1}\left(\gamma_{-}\right) \leq N, \quad c_{1}(\alpha) \leq N, \quad c_{1}(\beta) \leq N
$$

where $c_{1}(R)$ denotes the length of the first column of the Young diagram $R$.

The two-point function is diagonal under the free field computation

$$
\left\langle O_{A^{\prime}, i^{\prime} j^{\prime}}^{\gamma^{\prime}} O_{A, i j}^{\gamma}\right\rangle=m ! n ! \delta_{\gamma \gamma^{\prime}} \delta_{A A^{\prime}} \delta_{i i^{\prime}} \delta_{j j^{\prime}} d_{A} t^{\gamma},
$$

where the space-time dependence is omitted. $t^{\gamma}$ is the dimension of $\gamma$ considered as a representation of $U(N)$, and $d_{A}$ is the dimension of $A$ as a representation of $S_{m} \times S_{n}$.

If we regard the matrices $X$ and $Y$ as linear operators on a vector space $V$, the tensor product $X^{\otimes m} \otimes Y^{T \otimes n}$ can be regarded as a linear operator acting on the vector space $W=V^{\otimes m} \otimes \bar{V}^{\otimes n}$. The Brauer operator is constructed by acting with $Q_{A, i j}^{\gamma}$ on the tensor product $X^{\otimes m} \otimes Y^{T \otimes n}$ and taking a trace on $W$. The trace is written by $t r_{m, n}$. The $Q_{A, i j}^{\gamma}$ can be expressed as a linear combination of elements in the Brauer algebra, which can be written explicitly as

$$
Q_{A, i j}^{\gamma}=t^{\gamma} \sum_{b \in B_{N}(m, n)} \chi_{A, j i}^{\gamma}\left(b^{*}\right) b
$$

The coefficient of the linear combination is the restricted character of the Brauer algebra: $\chi_{A, j i}^{\gamma}(b)=$ $\chi^{\gamma}\left(Q_{A, i j}^{\gamma} b\right)$. Another ingredient appearing in $Q_{A, i j}^{\gamma}$ is $b^{*}$, which we call the dual element, is a specific linear combination of elements in the algebra. In [13] the following formula was given for the dual element:

$$
\Sigma\left(b_{i}^{*}\right)=\frac{1}{N^{m+n}} \Omega_{m+n}^{-1}\left(\Sigma\left(b_{i}\right)\right)^{-1},
$$

using a map $\Sigma: B_{N}(m, n) \rightarrow S_{m+n}$, and $\Omega_{m+n}$ is the Omega factor (see [13] for the definition). The index $i$ runs over a complete set of the elements in the algebra.

One important property of $Q_{A, i j}^{\gamma}$ is

$$
\sigma Q_{A, i j}^{\gamma} \sigma^{-1}=Q_{A, i j}^{\gamma}, \quad \sigma \in S_{m} \times S_{n}
$$


The projection operators of the Brauer algebra associated with an irreducible representation $\gamma$ are given by $P^{\gamma}=\sum_{A, i} Q_{A, i i}^{\gamma}$. Note that they are in the centre of the algebra. If we replace $X$ and $Y$ with a unitary matrix $U$ and the conjugate $U^{\dagger}, O^{\gamma}\left(U, U^{\dagger}\right)=t r_{m, n}\left(P^{\gamma} U^{\otimes m} \otimes U^{* \otimes n}\right)$ is the character of $U(N)$. So roughly speaking, our operators are obtained by decomposing the characters into some pieces.

We have the inverse formula

$$
t r_{m, n}\left(b X^{\otimes m} \otimes Y^{T \otimes n}\right)=\sum_{\gamma, A, i j} \frac{1}{d_{A}} \chi_{A, i j}^{\gamma}(b) t r_{m, n}\left(Q_{A, i j}^{\gamma} X^{\otimes m} \otimes Y^{T \otimes n}\right) .
$$

Noticing that any multi-trace operator can be written in the form of the left-hand side with a certain element $b$ of the algebra, this formula enables us to express any multi-trace operator in terms of the basis.

For an element $b_{0}$ satisfying $\sigma b_{0}=b_{0} \sigma$ for any $\sigma \in S_{m} \times S_{n}$, we have (see appendix B in [17])

$$
b_{0} Q_{A, i j}^{\gamma}=\frac{1}{d_{A}} \sum_{k} \chi_{A, k i}^{\gamma}\left(b_{0}\right) Q_{A, k j}^{\gamma} .
$$

This is consistent with the inverse formula. Making use of this formula, we can derive the following

$$
\begin{aligned}
\sum_{A, k l} \frac{1}{d_{A}} \chi_{A, k l}^{\gamma}\left(b_{0}\right) \chi_{A, l k}^{\gamma}(b) & =\sum_{A, k l} \frac{1}{d_{A}} \chi_{A, k l}^{\gamma}\left(b_{0}\right) \chi^{\gamma}\left(Q_{A, k l}^{\gamma} b\right) \\
& =\sum_{A, l} \chi^{\gamma}\left(b_{0} Q_{A, l l}^{\gamma} b\right) \\
& =\chi^{\gamma}\left(b_{0} b\right) .
\end{aligned}
$$

The above things are for the $\mathrm{su}(2)$ sector, but it is also possible to accommodate more than two fields [40. For example, the basis for $m-s X^{\prime}$ 's, $s W_{1}$ 's, $n-t Y$ 's and $t W_{2}$ 's is given by the same way just by replacing $A$ with $r$ that is an irreducible representation of $S_{m-s} \times S_{s} \times S_{n-t} \times S_{t}$. The inverse formula for this case is

$$
\begin{gathered}
t r_{m, n}\left(b X^{\otimes m-s} \otimes W_{1}^{\otimes s} \otimes Y^{T \otimes n-t} \otimes W_{2}^{T \otimes t}\right) \\
=\sum_{\gamma, r, i j} \frac{1}{d_{r}} \chi_{r, i j}^{\gamma}(b) t r_{m, n}\left(Q_{r, i j}^{\gamma} X^{\otimes m-s} \otimes W_{1}^{\otimes s} \otimes Y^{T \otimes n-t} \otimes W_{2}^{T \otimes t}\right) .
\end{gathered}
$$

We also have

$$
\sum_{r, k l} \frac{1}{d_{r}} \chi_{r, k l}^{\gamma}\left(b_{0}\right) \chi_{r, l k}^{\gamma}(b)=\chi^{\gamma}\left(b_{0} b\right)
$$

where $b_{0}$ is an element that commutes with any elements in $S_{m-s} \times S_{s} \times S_{n-t} \times S_{t}$.

\section{B A reduction formula}

In this appendix, we will give a formula associated with the restriction from $B_{N}(m, n)$ to $B_{N}(m, n-$ 1). We will embed the subalgebra into $B_{N}(m, n)$ by removing the last slot of the $(m+n)$ slots. 
Under the embedding the elements of $B_{N}(m, n)$ can be expressed in terms of the elements of the subalgebra $B_{N}(m, n-1)$ as

$$
b_{i}=\left\{c_{a},(\bar{j} \bar{n}) c_{a}, c_{a} C_{k, n}\right\}
$$

where the index $i$ runs over a basis of $B_{N}(m, n), i=1, \cdots,(m+n)$ !, and $j=1, \cdots, n-1$ and $k=1, \cdots, m$. We have denoted the elements of $B_{N}(m, n-1)$ by $c_{a}$, where $a=1, \cdots,(m+n-1) !$.

We denote the dual element in $B_{N}(m, n)$ by $b^{*}$ and the dual element in $B_{N}(m, n-1)$ by $c^{*}$, which are defined by

$$
\Sigma\left(b_{i}^{*}\right)=\frac{1}{N^{m+n}} \Omega_{m+n}^{-1}\left(\Sigma\left(b_{i}\right)\right)^{-1},
$$

and

$$
\Sigma\left(c_{a}^{\tilde{*}}\right)=\frac{1}{N^{m+n-1}} \Omega_{m+n-1}^{-1}\left(\Sigma\left(c_{a}\right)\right)^{-1} .
$$

Note that $\Sigma$ is a map from $B_{N}(m, n)$ to $S_{m+n}$.

Using (B.2) for $b_{i}=c_{a}$, we get

$$
\begin{aligned}
\Sigma\left(c_{a}^{*}\right) & =\frac{1}{N^{m+n}} \Omega_{m+n}^{-1}\left(\Sigma\left(c_{a}\right)\right)^{-1} \\
& =\frac{1}{N} \Omega_{m+n}^{-1} \Omega_{m+n-1} \Sigma\left(c_{a}^{\tilde{*}}\right) .
\end{aligned}
$$

In the last step we have used (B.3). Note that $\Omega_{m+n-1}$ is the Omega factor defined in $S_{m+n-1}$ embedded in $S_{m+n}$ by removing the $(m+n)$-th slot.

Similarly, for $b_{i}=(\bar{j} \bar{n}) c_{a}$, we have

$$
\begin{aligned}
\Sigma\left(\left((\bar{j} \bar{n}) c_{a}\right)^{*}\right) & =\frac{1}{N^{m+n}} \Omega_{m+n}^{-1}\left(\Sigma\left((\bar{j} \bar{n}) c_{a}\right)\right)^{-1} \\
& =\frac{1}{N^{m+n}} \Omega_{m+n}^{-1}\left(\Sigma\left(c_{a}\right)(j n)\right)^{-1} \\
& =\frac{1}{N^{m+n}} \Omega_{m+n}^{-1}(j n)\left(\Sigma\left(c_{a}\right)\right)^{-1} \\
& =\frac{1}{N}(j n) \Omega_{m+n}^{-1} \Omega_{m+n-1} \Sigma\left(c_{a}^{*}\right),
\end{aligned}
$$

and for $b_{i}=c_{a} C_{k n}$ we have

$$
\begin{aligned}
\Sigma\left(\left(c_{a} C_{k n}\right)^{*}\right) & =\frac{1}{N^{m+n}} \Omega_{m+n}^{-1}\left(\Sigma\left(c_{a} C_{k n}\right)\right)^{-1} \\
& =\frac{1}{N^{m+n}} \Omega_{m+n}^{-1}\left(\Sigma\left(c_{a}\right)(k n)\right)^{-1} \\
& =\frac{1}{N^{m+n}} \Omega_{m+n}^{-1}(k n)\left(\Sigma\left(c_{a}\right)\right)^{-1} \\
& =\frac{1}{N}(k n) \Omega_{m+n}^{-1} \Omega_{m+n-1} \Sigma\left(c_{a}^{\tilde{*}}\right) .
\end{aligned}
$$

Note that we have used the following properties that come from the definition of $\Sigma$,

$$
\begin{aligned}
& \Sigma\left(c_{a} C_{k n}\right)=\Sigma\left(c_{a}\right)(k n), \\
& \Sigma\left((\bar{j} \bar{n}) c_{a}\right)=\Sigma\left(c_{a}\right)(j n) .
\end{aligned}
$$


Now combining (․․4 $),(\underline{\mathrm{B} .5})$ and $(\underline{\mathrm{B} .6})$, for $O_{c_{a}}^{*}:=N c_{a}^{*}+\sum_{j=1}^{n-1}\left((\bar{j} \bar{n}) c_{a}\right)^{*}+\sum_{k=1}^{m}\left(c_{a} C_{k n}\right)^{*}$ we have an interesting equation: 7

$$
\begin{aligned}
\Sigma\left(O_{c_{a}}^{*}\right) & =\left(1+\frac{1}{N} \sum_{i \neq n}(i n)\right) \Omega_{m+n}^{-1} \Omega_{m+n-1}\left(\Sigma\left(c_{a}^{\tilde{*}}\right)\right) \\
& =\Sigma\left(c_{a}^{\tilde{*}}\right)
\end{aligned}
$$

which also means

$$
O_{c_{a}}^{*}=c_{a}^{\tilde{*}}
$$

Note that $c_{a}^{*}$ is not an element in $B_{N}(m, n-1)$, but $c_{a}^{\tilde{*}}$ is an element in $B_{N}(m, n-1)$.

For the reduction $B_{N}(2,1) \rightarrow B_{N}(1,1)$, we can confirm the following equation by computing the dual element explicitly:

$$
N 1^{*}+(12)^{*}+C_{21}^{*}=\frac{1}{N^{2}-1}\left(1-\frac{C_{11}}{N}\right)=1^{\tilde{*}}
$$

where $1^{*}$ is defined in $B_{N}(1,1)$. We can also find explicitly that the formula is valid in the reduction $B_{N}(2,1) \rightarrow S_{2}$ :

$$
N 1^{*}+C_{11}^{*}+C_{21}^{*}=\frac{1}{N^{2}-1}\left(1-\frac{s}{N}\right)=\frac{1}{N^{2}+N s}=\frac{1}{N^{2} \Omega_{2}}=1^{\tilde{*}},
$$

where $1^{\tilde{*}}$ is defined in $S_{2}$.

\section{Derivation of (30)}

In this appendix we present explicit calculation to derive (30). The first term in (29) is

$$
\begin{aligned}
& \operatorname{tr}\left(:\left[[Y, X], \partial_{X}\right]\left[\left[\partial_{Y}, \partial_{X}\right], X\right]:\right) \\
= & \operatorname{tr}\left(:[Y, X] \partial_{X}\left[\partial_{Y}, \partial_{X}\right] X:\right)-\operatorname{tr}\left(:[Y, X] \partial_{X} X\left[\partial_{Y}, \partial_{X}\right]:\right) \\
& -\operatorname{tr}\left(: \partial_{X}[Y, X]\left[\partial_{Y}, \partial_{X}\right] X:\right)+\operatorname{tr}\left(: \partial_{X}[Y, X] X\left[\partial_{Y}, \partial_{X}\right]:\right) .
\end{aligned}
$$

In what follows we will not write the normal ordering symbol explicitly. Using $\left(\partial_{X}\right)_{j}^{i} X_{l}^{k}=\delta_{l}^{i} \delta_{j}^{k}$,

$$
\begin{aligned}
& \operatorname{tr}\left(A \partial_{X} \partial_{Y} \partial_{X}\right) O_{A, i j}^{\gamma}(X, Y) \\
= & (A)_{b}^{a}\left(\partial_{X}\right)_{c}^{b}\left(\partial_{Y}\right)_{d}^{c}\left(\partial_{X}\right)_{a}^{d}\left(Q_{A, i j}^{\gamma}\right)_{I K}^{J L} X_{J}^{I}\left(Y^{T}\right)_{L}^{K} \\
= & m(m-1) n(A)_{b}^{a}\left(Q_{A, i j}^{\gamma}\right)_{a c j_{3} \cdots c k_{2} \cdots}^{d b j_{3} \cdots} X_{j_{3} \cdots}^{i_{3} \cdots}\left(Y^{T}\right)_{l_{2} \cdots}^{k_{2} \cdots} \\
= & m(m-1) n t r_{m, n}\left(Q_{A, i j}^{\gamma}(12) C_{1,1} 1 \otimes A \otimes X^{\otimes m-2} \otimes 1 \otimes Y^{T \otimes n-1}\right) \\
= & m(m-1) n t r_{m, n}\left(C_{1,1}(12) Q_{A, i j}^{\gamma} 1 \otimes A \otimes X^{\otimes m-2} \otimes 1 \otimes Y^{T \otimes n-1}\right),
\end{aligned}
$$

\footnotetext{
${ }^{7}$ Note the following
}

$$
\left(1+\frac{1}{N} \sum_{i \neq n}(i n)\right) \Omega_{m+n-1}=\Omega_{m+n},
$$

which appeared in 44. It was also exploited in 13 . 
where $A=X[Y, X]$. Likewise, we have

$$
\begin{aligned}
& \operatorname{tr}\left(A \partial_{X} \partial_{X} \partial_{Y}\right) O_{A, i j}^{\gamma}(X, Y) \\
= & m(m-1) n(A)_{b}^{a}\left(Q_{A, i j}^{\gamma}\right)_{d c i_{3} \cdots d k_{2} \cdots}^{c b j_{3} \cdots a l_{2} \cdots} X_{j_{3} \cdots}^{i_{3} \cdots}\left(Y^{T}\right)_{l_{2} \cdots}^{k_{2} \cdots} \\
= & m(m-1) n t r_{m, n}\left(Q_{A, i j}^{\gamma} C_{1,1}(12) 1 \otimes A \otimes X^{\otimes m-2} \otimes 1 \otimes Y^{T \otimes n-1}\right) .
\end{aligned}
$$

Then one finds that the first line in (C.1) can be expressed by

$$
m(m-1) n t r_{m, n}\left(\left[C_{1,1}(12), Q_{A, i j}^{\gamma}\right] 1 \otimes X[Y, X] \otimes X^{\otimes m-2} \otimes 1 \otimes Y^{T \otimes n-1}\right) .
$$

We next calculate

$$
\begin{aligned}
& \operatorname{tr}\left(B \partial_{X} X \partial_{Y} \partial_{X}\right) O_{A, i j}^{\gamma}(X, Y) \\
= & (B)_{b}^{a}\left(\partial_{X}\right)_{c}^{b}(X)_{d}^{c}\left(\partial_{Y}\right)_{e}^{d}\left(\partial_{X}\right)_{a}^{e}\left(Q_{A, i j}^{\gamma}\right)_{I K}^{J L} X_{J}^{I}\left(Y^{T}\right)_{L}^{K} \\
= & m(m-1) n(B)_{b}^{a}(X)_{d}^{c}\left(Q_{A, i j}^{\gamma}\right)_{a c i_{3} \cdots d k_{2} \cdots}^{e b j_{3} \cdots e l_{2} \cdots} X_{j_{3} \cdots}^{i_{3} \cdots}\left(Y^{T}\right)_{l_{2} \cdots}^{k_{2} \cdots} \\
= & m(m-1) n t r_{m, n}\left((12) C_{1,1} Q_{A, i j}^{\gamma} B \otimes X^{\otimes m-1} \otimes 1 \otimes Y^{T \otimes n-1}\right),
\end{aligned}
$$

where $B=[Y, X]$, and

$$
\begin{aligned}
& \operatorname{tr}\left(B \partial_{X} X \partial_{X} \partial_{Y}\right) O_{A, i j}^{\gamma}(X, Y) \\
= & m(m-1) n(B)_{b}^{a}(X)_{d}^{c}\left(Q_{A, i j}^{\gamma}\right)_{e c i_{3} \cdots e k_{2} \cdots}^{d b j_{3} \cdots a l_{2} \cdots} X_{j_{3} \cdots}^{i_{3} \cdots}\left(Y^{T}\right)_{l_{2} \cdots}^{k_{2} \cdots} \\
= & m(m-1) n t r_{m, n}\left(Q_{A, i j}^{\gamma}(12) C_{1,1} B \otimes X^{\otimes m-1} \otimes 1 \otimes Y^{T \otimes n-1}\right) .
\end{aligned}
$$

From these two, one finds that the second term in (C.1) can be expressed by

$$
-m(m-1) n t r_{m, n}\left(\left[(12) C_{1,1}, Q_{A, i j}^{\gamma}\right] B \otimes X^{\otimes m-1} \otimes 1 \otimes Y^{T \otimes n-1}\right) .
$$

Similarly, we have

$$
\begin{aligned}
& \operatorname{tr}\left(B \partial_{Y} \partial_{X} X \partial_{X}\right) O_{A, i j}^{\gamma}(X, Y) \\
= & m(m-1) n(B)_{b}^{a}(X)_{e}^{d}\left(Q_{A, i j}^{\gamma}\right)_{a d i_{3} \cdots b k_{2} \cdots}^{e c j_{3} \cdots c l_{2} \cdots} X_{j_{3} \cdots}^{i_{3} \cdots}\left(Y^{T}\right)_{l_{2} \cdots}^{k_{2} \cdots} \\
= & m(m-1) n t r_{m, n}\left(C_{1,1}(12) Q_{A, i j}^{\gamma} B \otimes X^{\otimes m-1} \otimes 1 \otimes Y^{T \otimes n-1}\right),
\end{aligned}
$$

and

$$
\begin{aligned}
& \operatorname{tr}\left(B \partial_{X} \partial_{Y} X \partial_{X}\right) O_{A, i j}^{\gamma}(X, Y) \\
= & m(m-1) n(B)_{b}^{a}(X)_{e}^{d}\left(Q_{A, i j}^{\gamma}\right)_{a c i_{3} \cdots c k_{2} \cdots}^{e b j_{3} \cdots d l_{2} \cdots} X_{j_{3} \cdots}^{i_{3} \cdots}\left(Y^{T}\right)_{l_{2} \cdots}^{k_{2} \cdots} \\
= & m(m-1) n t r_{m, n}\left(Q_{A, i j}^{\gamma} C_{1,1}(12) B \otimes X^{\otimes m-1} \otimes 1 \otimes Y^{T \otimes n-1}\right) .
\end{aligned}
$$

One then finds that the third term in (C.1) can be expressed by

$$
-m(m-1) n t r_{m, n}\left(\left[C_{1,1}(12), Q_{A, i j}^{\gamma}\right] B \otimes X^{\otimes m-1} \otimes 1 \otimes Y^{T \otimes n-1}\right) .
$$

Finally we have

$$
\begin{aligned}
& \operatorname{tr}\left(\tilde{A} \partial_{Y} \partial_{X} \partial_{X}\right) O_{A, i j}^{\gamma}(X, Y) \\
= & m(m-1) n(\tilde{A})_{b}^{a}\left(Q_{A, i j}^{\gamma}\right)_{d a i_{3} \cdots b k_{2} \cdots}^{c d j_{3} \cdots c l_{2} \cdots} X_{j_{3} \cdots}^{i_{3} \cdots}\left(Y^{T}\right)_{l_{2} \cdots}^{k_{2} \cdots} \\
= & m(m-1) n t r_{m, n}\left((12) C_{1,1} Q_{A, i j}^{\gamma} 1 \otimes \tilde{A} \otimes X^{\otimes m-2} \otimes 1 \otimes Y^{T \otimes n-1}\right),
\end{aligned}
$$


where $\tilde{A}=[Y, X] X$, and

$$
\begin{aligned}
& \operatorname{tr}\left(\tilde{A} \partial_{X} \partial_{Y} \partial_{X}\right) O_{A, i j}^{\gamma}(X, Y) \\
= & m(m-1) n t r_{m, n}\left(Q_{A, i j}^{\gamma}(12) C_{11} 1 \otimes \tilde{A} \otimes X^{\otimes m-2} \otimes 1 \otimes Y^{T \otimes n-1}\right)
\end{aligned}
$$

to give the following expression for the last term in (C.1):

$$
m(m-1) n t r_{m, n}\left(\left[(12) C_{1,1}, Q_{A, i j}^{\gamma}\right] 1 \otimes \tilde{A} \otimes X^{\otimes m-2} \otimes 1 \otimes Y^{T \otimes n-1}\right) .
$$

\section{References}

[1] J. A. Minahan and K. Zarembo, "The Bethe ansatz for N=4 superYang-Mills," JHEP 0303 (2003) 013 hep-th/0212208.

[2] N. Beisert, C. Kristjansen and M. Staudacher, "The Dilatation operator of conformal N=4 superYang-Mills theory," Nucl. Phys. B 664 (2003) 131 hep-th/0303060.

[3] A. V. Ryzhov, "Quarter BPS operators in N = 4 SYM," JHEP 0111 (2001) 046 arXiv:hep-th/0109064.

[4] M. Bianchi, B. Eden, G. Rossi and Y. S. Stanev, "On operator mixing in N = 4 SYM," Nucl. Phys. B 646 (2002) 69 [arXiv:hep-th/0205321]; M. Bianchi, G. Rossi and Y. S. Stanev, "Surprises from the resolution of operator mixing in N = 4 SYM," Nucl. Phys. B 685 (2004) 65 arXiv:hep-th/0312228.

[5] G. Arutyunov, S. Penati, A. C. Petkou, A. Santambrogio and E. Sokatchev, "Non-protected operators in N = 4 SYM and multiparticle states of AdS(5) SUGRA," Nucl. Phys. B 643 (2002) 49 arXiv:hep-th/0206020.

[6] B. Eden, "On two fermion BMN operators," Nucl. Phys. B 681 (2004) 195 arXiv:hep-th/0307081.

[7] N. Beisert et al., "Review of AdS/CFT Integrability: An Overview," Lett. Math. Phys. 99 (2012) 3 [arXiv:1012.3982 [hep-th]].

[8] S. Corley, A. Jevicki and S. Ramgoolam, "Exact correlators of giant gravitons from dual N=4 SYM theory," Adv. Theor. Math. Phys. 5 (2002) 809 hep-th/0111222]; S. Corley and S. Ramgoolam, "Finite factorization equations and sum rules for BPS correlators in $\mathrm{N}=4$ SYM theory," Nucl. Phys. B 641 (2002) 131 [hep-th/0205221].

[9] V. Balasubramanian, M. Berkooz, A. Naqvi and M. J. Strassler, "Giant gravitons in conformal field theory," JHEP 0204 (2002) 034 hep-th/0107119.

[10] J. McGreevy, L. Susskind and N. Toumbas, "Invasion of the giant gravitons from Anti-de Sitter space," JHEP 0006 (2000) 008 hep-th/0003075.

[11] D. Berenstein, "A Toy model for the AdS / CFT correspondence," JHEP 0407 (2004) 018 hep-th/0403110.

[12] H. Lin, O. Lunin and J. M. Maldacena, "Bubbling AdS space and 1/2 BPS geometries," JHEP 0410 (2004) 025 hep-th/0409174. 
[13] Y. Kimura and S. Ramgoolam, "Branes, anti-branes and brauer algebras in gauge-gravity duality," JHEP 0711 (2007) 078 [arXiv:0709.2158 [hep-th]].

[14] T. W. Brown, P. J. Heslop and S. Ramgoolam, "Diagonal multi-matrix correlators and BPS operators in N=4 SYM," JHEP 0802 (2008) 030 arXiv:0711.0176 [hep-th]]; T. W. Brown, P. J. Heslop and S. Ramgoolam, "Diagonal free field matrix correlators, global symmetries and giant gravitons," JHEP 0904 (2009) 089 [arXiv:0806.1911 [hep-th]].

[15] R. Bhattacharyya, S. Collins and R. d. M. Koch, "Exact Multi-Matrix Correlators," JHEP 0803 (2008) 044 [arXiv:0801.2061 [hep-th]].

[16] R. Bhattacharyya, R. de Mello Koch and M. Stephanou, "Exact Multi-Restricted Schur Polynomial Correlators," JHEP 0806 (2008) 101 [arXiv:0805.3025 [hep-th]].

[17] Y. Kimura and S. Ramgoolam, "Enhanced symmetries of gauge theory and resolving the spectrum of local operators," Phys. Rev. D 78 (2008) 126003 [arXiv:0807.3696 [hep-th]].

[18] R. de Mello Koch, J. Smolic and M. Smolic, "Giant Gravitons - with Strings Attached (I)," JHEP 0706 (2007) 074 hep-th/0701066; R. de Mello Koch, J. Smolic and M. Smolic, "Giant Gravitons - with Strings Attached (II)," JHEP 0709 (2007) 049 [hep-th/0701067].

[19] D. Bekker, R. de Mello Koch and M. Stephanou, "Giant Gravitons - with Strings Attached. III.," JHEP 0802 (2008) 029 arXiv:0710.5372 [hep-th]].

[20] T. W. Brown, "Permutations and the Loop," JHEP 0806 (2008) 008 arXiv:0801.2094 [hepth]].

[21] T. W. Brown, "Cut-and-join operators and N=4 super Yang-Mills," JHEP 1005 (2010) 058 [arXiv:1002.2099 [hep-th]].

[22] Y. Kimura, "Quarter BPS classified by Brauer algebra," JHEP 1005 (2010) 103 [arXiv:1002.2424 [hep-th]].

[23] R. d. M. Koch, G. Mashile and N. Park, "Emergent Threebrane Lattices," Phys. Rev. D 81 (2010) 106009 arXiv:1004.1108 [hep-th]].

[24] J. Pasukonis and S. Ramgoolam, "From counting to construction of BPS states in N=4 SYM," JHEP 1102 (2011) 078 [arXiv:1010.1683 [hep-th]].

[25] V. De Comarmond, R. de Mello Koch and K. Jefferies, "Surprisingly Simple Spectra," JHEP 1102 (2011) 006 [arXiv:1012.3884 [hep-th]].

[26] W. Carlson, R. d. M. Koch and H. Lin, "Nonplanar Integrability," JHEP 1103 (2011) 105 arXiv:1101.5404 [hep-th]]; R. d. M. Koch, B. A. E. Mohammed and S. Smith, "Nonplanar Integrability: Beyond the SU(2) Sector," arXiv:1106.2483 [hep-th]; R. de Mello Koch, P. Diaz and H. Soltanpanahi, "Non-planar Anomalous Dimensions in the sl(2) Sector," arXiv:1111.6385 [hep-th]; R. de Mello Koch, B. A. E. Mohammed, J. Murugan and A. Prinsloo, "Beyond the Planar Limit in ABJM," JHEP 1205 (2012) 037 arXiv:1202.4925 [hep-th]]; R. d. M. Koch, P. Diaz and N. Nokwara, "Restricted Schur Polynomials for Fermions and integrability in the su(2|3) sector," arXiv:1212.5935 [hep-th]. 
[27] R. d. M. Koch, M. Dessein, D. Giataganas and C. Mathwin, "Giant Graviton Oscillators," JHEP 1110 (2011) 009 [arXiv:1108.2761 [hep-th]].

[28] R. de Mello Koch, G. Kemp and S. Smith, "From Large N Nonplanar Anomalous Dimensions to Open Spring Theory," Phys. Lett. B 711 (2012) 398 [arXiv:1111.1058 [hep-th]].

[29] R. d. M. Koch and S. Ramgoolam, "A double coset ansatz for integrability in AdS/CFT," arXiv:1204.2153 [hep-th].

[30] R. de Mello Koch, G. Kemp, B. A. E. Mohammed and S. Smith, "Nonplanar integrability at two loops," JHEP 1210 (2012) 144 [arXiv:1206.0813 [hep-th]].

[31] B. A. E. Mohammed, "Nonplanar Integrability and Parity in ABJ Theory," arXiv:1207.6948 [hep-th].

[32] R. d. M. Koch, J. Murugan and N. Nokwara, "Large N anomalous dimensions for large operators in Leigh-Strassler deformed SYM," arXiv:1212.6624 [hep-th].

[33] S. Bellucci, P. Y. Casteill, J. F. Morales and C. Sochichiu, "Spin bit models from non-planar N = 4 SYM," Nucl. Phys. B 699 (2004) 151 arXiv:hep-th/0404066];

[34] P. -Y. Casteill, R. A. Janik, A. Jarosz and C. Kristjansen, "Quasilocality of joining/splitting strings from coherent states," JHEP 0712 (2007) 069 [arXiv:0710.4166 [hep-th]].

[35] C. Kristjansen, "Review of AdS/CFT Integrability, Chapter IV.1: Aspects of Non-Planarity," Lett. Math. Phys. 99 (2012) 349 [arXiv:1012.3997] [hep-th]].

[36] J. R. Stembridge, "Rational tableaux and the tensor algebra of $g l(n), \mathrm{h}$ J. Combin.Theory A 46 (1987) 79.

[37] K. Koike, "On the Decomposition of Tensor Products of the Representations of the Classical Groups: By Means of the Universal Characters," Advances in Mathematics 74 (1989) 57-86.

[38] M. Benkart, M. Chakrabarti, T. Halverson, C. Lee, R. Leduc and J. Stroomer, "Tensor product representations of general linear groups and their connections with Brauer algebras, $\mathrm{h}$ J. Algebra, 166 (1994), 529.

[39] Y. Kimura and H. Lin, "Young diagrams, Brauer algebras, and bubbling geometries," JHEP 1201 (2012) 121 arXiv:1109.2585 [hep-th]].

[40] Y. Kimura, "Correlation functions and representation bases in free N=4 Super Yang-Mills," Nucl. Phys. B 865 (2012) 568 arXiv:1206.4844 [hep-th]].

[41] Y. Kimura, "Non-holomorphic multi-matrix gauge invariant operators based on Brauer algebra," JHEP 0912 (2009) 044 arXiv:0910.2170 [hep-th]].

[42] D. Berenstein, "Giant gravitons: a collective coordinate approach," arXiv:1301.3519 [hep-th].

[43] Y. Kimura, S. Ramgoolam and D. Turton, "Free particles from Brauer algebras in complex matrix models," JHEP 1005 (2010) 052 [arXiv:0911.4408 [hep-th]].

[44] S. Ramgoolam, "Wilson loops in 2-D Yang-Mills: Euler characters and loop equations," Int. J. Mod. Phys. A 11 (1996) 3885 hep-th/9412110. 